\title{
Optical tracking of a nanoparticle trapped by a double nanohole aperture
}

\author{
Zhe $\mathrm{Xu}^{1}$, Wuzhou Song ${ }^{1}$, and Kenneth B. Crozier ${ }^{1,2}$ \\ School of Physics, University of Melbourne, Victoria 3010, Australia \\ ${ }^{2}$ Department of Electrical and Electronic Engineering, University of Melbourne, Victoria 3010, Australia \\ Corresponding Author e-mail address: kenneth.crozier@unimelb.edu.au
}

\begin{abstract}
A double nanohole aperture in a gold film is used to trap a polytyrene nanosphere with a diameter of $20 \mathrm{~nm}$. Fluorescence microscopy is used to track the position of the trapped nanosphere.

OCIS codes: (140.7010) Laser trapping; (240.6680) Surface plasmons;
\end{abstract}

\section{Introduction}

Conventional optical tweezers trap particles using the forces exerted by laser beams focused with lenses. They face challenges for the trapping of very small particles however because the forces are roughly proportional to particle volume. This means that the field intensity must be increased to maintain trap stability, but this can lead to deleterious thermal effects. In addition, diffraction sets a lower bound on the focal spot size, thereby determining the precision with which an object can be trapped. This has motivated interest in optical traps based on plasmonics [1]. Of note has been the 'self-induced back action' (SIBA) effect, in which the position of a trapped particle alters the resonance of a nanophotonic structure such as a nanoaperture [2]. It has been argued that using the SIBA effect, nanoparticles (NPs) can be stably trapped with laser powers and local intensities much smaller than what would be required using conventional tweezers [2] and experimental demonstrations have been made [3]. Recent theoretical analysis [4] has furthermore suggested that SIBA could enable a remarkable range of optical manipulation capabilities beyond those already demonstrated. The understanding and further development of the SIBA effect would be greatly facilitated by direct observation of the dynamics of trapped nanoparticles, i.e. position as a function of time. To the best of our knowledge, however, this has not been demonstrated in previous reports (e.g. [3]). In these reports, the trapping process was monitored by measuring the optical transmission (of trapping laser or of fluorescence from the trapped object) through the nanoaperture as a function of time, with step-like increases in transmission indicating the trapping of a nanoparticle. Here, we employ fluorescence microscopy to track the position of a polystyrene nanosphere (20 nm diameter) trapped via the SIBA effect by a double nanohole (DNH) aperture in a gold film. We anticipate that our method will be valuable tool for the development of the SIBA effect.

\section{Electromagnetic simulations}

We first present electromagnetic simulations of the force exerted on a nanoparticle near a DNH aperture (Figure 1).
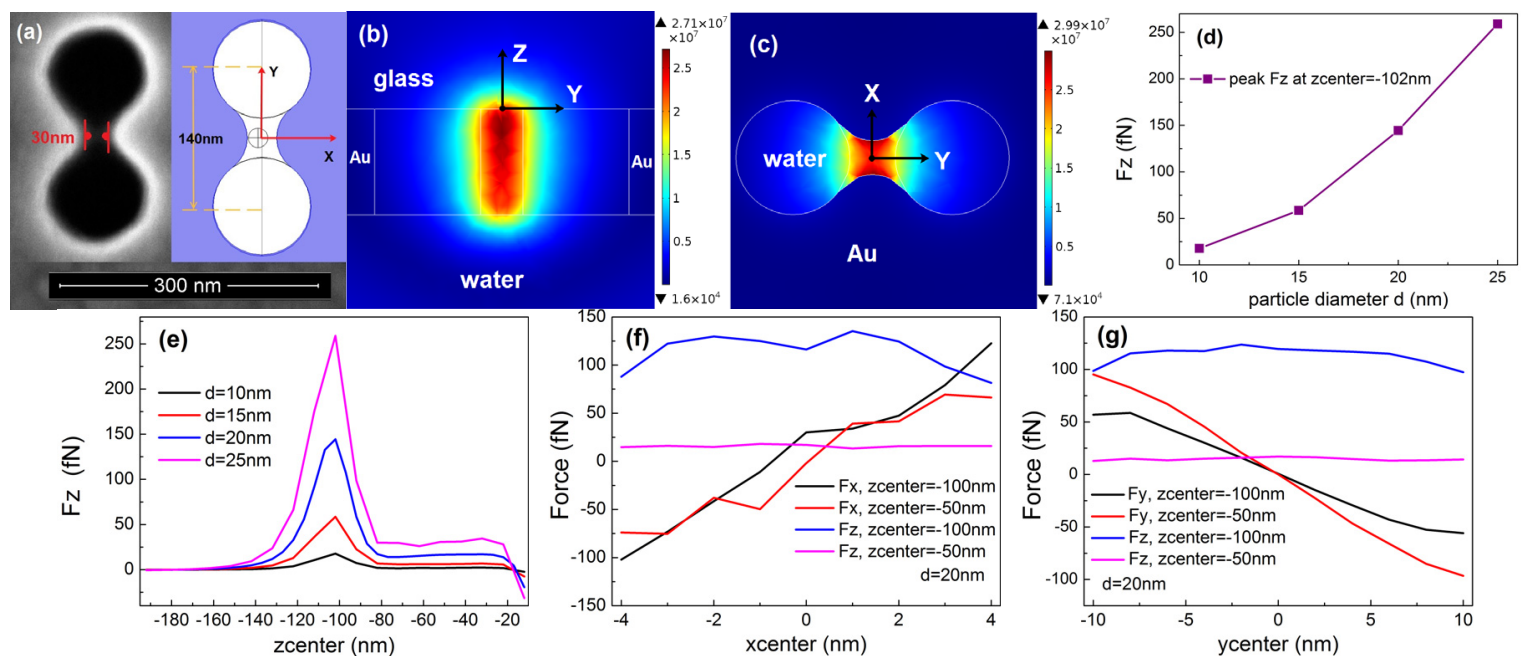

Fig.1. (a) DNH aperture in Au film: LEFT-scanning electron micrograph (SEM), RIGHT-simulated structure, comprising two holes (diameter $D$ $=100 \mathrm{~nm}$ ) separated by $140 \mathrm{~nm}$ (center-to-center). (b), (c) Simulation electric field (|E|) distribution, for plane wave illumination from water side (traveling in $+z$ direction, intensity $I=6.67 \mathrm{~mW} / \mu \mathrm{m}^{2}$ ). Black dot indicates coordinate system origin. (b) $x=0$ cross section $(\mathrm{c}) z=-50 \mathrm{~nm}$ cross section. (d),(e),(f),(g) Force exerted on particle $(n=1.6)$ as a function of particle diameter $d$ and position. For intensity $I=6.67 \mathrm{~mW} / \mu \mathrm{m}^{2}$. 
We use COMSOL to simulate our DNH (Fig.1a), formed in a gold film (100 nm thick) on a glass substrate covered with water. Plane wave illumination ( $x$-polarized, $\lambda=1064 \mathrm{~nm}$ ) is incident from the water side, resulting in highly confined electric fields in the DNH (Fig.1b-c, no NP present). We next introduce our NP, compute the electric and magnetic fields on a surface that surrounds it, and determine the force using the Maxwell stress tensor (MST). In Fig.1d, we plot the peak force in the $z$-direction $F_{z}$ as a function of NP diameter, assuming that the NP center is at $\left(x_{\text {center }}, y_{\text {center }}, z_{\text {center }}\right)=(0,0,-102 \mathrm{~nm})$. It can be seen that force increases with diameter $d$. In Fig.1e, we plot $F_{z}$ as a function of NP position along the $z$-axis, for $\left(x_{\text {center }}, y_{\text {center }}\right)=(0,0)$. It can be seen that the NP is pulled into the DNH ( $F_{z}$ is positive). In Fig.1f, we plot $F_{x}$ and $F_{z}$ as functions of NP position along the $x$-axis, with $y_{c e n t e r}=0 \mathrm{~nm}$, and $z_{\text {center }}=-50$ or $-100 \mathrm{~nm}$. The positive values of $F_{z}$ confirm that the NP is pulled into the DNH. It can also be seen that $F_{x}$ is positive (negative) when $x_{\text {center }}$ is positive (negative). This indicates that the NP is pulled to the sides of the DNH gap (30 nm wide), to the positions denoted by red dots in Fig.1a. In Fig.1g, we plot $F_{y}$ and $F_{z}$ as functions of NP position along the $y$-axis, with $x_{\text {center }}=0 \mathrm{~nm}$, and $z_{\text {center }}=-50$ or $-100 \mathrm{~nm}$. The positive values of $F_{z}$ again confirm that the NP is pulled into the DNH. It can also be seen that $F_{y}$ is positive (negative) when $y_{\text {center }}$ is negative (positive). This indicates that the NP experiences a restoring force for excursions in the $y$-direction from the DNH center $(y=0)$.

\section{Experimental results: dynamics of a trapped nanoparticle}

Fig. 2a schematically illustrates our set-up. An $x$-polarized laser beam $(\lambda=1064 \mathrm{~nm})$ is focused by an oil immersion objective (100×, NA 1.3) into a sample chamber containing water, polystyrene NPs (FluoSpheres, 20 nm diameter, carboxylate-modified, Nile Red, Life Technologies) and a trace amount of surfactant (Tween 20) to prevent aggregation. A green laser $(\lambda=532 \mathrm{~nm})$ excites fluorescence, which is imaged onto an electron multiplying camera (EM-CCD) operated at 30 fps. Several EM-CCD frames are shown as Fig. 2b-d. At time t0, the NP moves randomly and is $\sim 2.8 \mu \mathrm{m}$ from the DNH. From time t1, the NP is stably trapped for $\sim 34$ seconds. At time t2, it is no longer trapped. We verify that blocking the laser results in quick release of the NP from the trap. We also verify that the NP is less strongly trapped (larger positional fluctuations) when the laser power is reduced or the polarization is rotated. To illustrate the trapping process, we plot the fluorescence intensity integrated over a $30 \times 30$ pixel cross section centered over the DNH vs time. At $\mathrm{t} \sim 6 \mathrm{~s}$, the NP enters the DNH vicinity and becomes trapped, resulting in an abrupt jump in fluorescence. At $\mathrm{t} \sim 49 \mathrm{~s}$, the trapping laser is turned off and the NP is released. Turning the laser on at $\mathrm{t} \sim 55 \mathrm{~s}$ results in the NP being trapped again. To assess the trapping more quantitatively, we determine the NP center positions from 1000 EM-CCD frames using a radial-symmetry-based particle localization algorithm [5] over an interval of 33s (Fig.2f). In Fig.2g-h, we plot the positions ( $x_{c}$ and $y_{c}$ ) as histograms. The full-widths-at-half maximum (FWHMs) of fitted Gaussian distributions are 59 and $190 \mathrm{~nm}$ along the $x$ - and $y$-directions, respectively.

Fabrication was performed by G. Gervinskas (Melbourne Centre for Nanofabrication) of the Australian Nanofabrication Network (ANFF). Funding: Laby Foundation, VESKI, and the Australian Research Council.
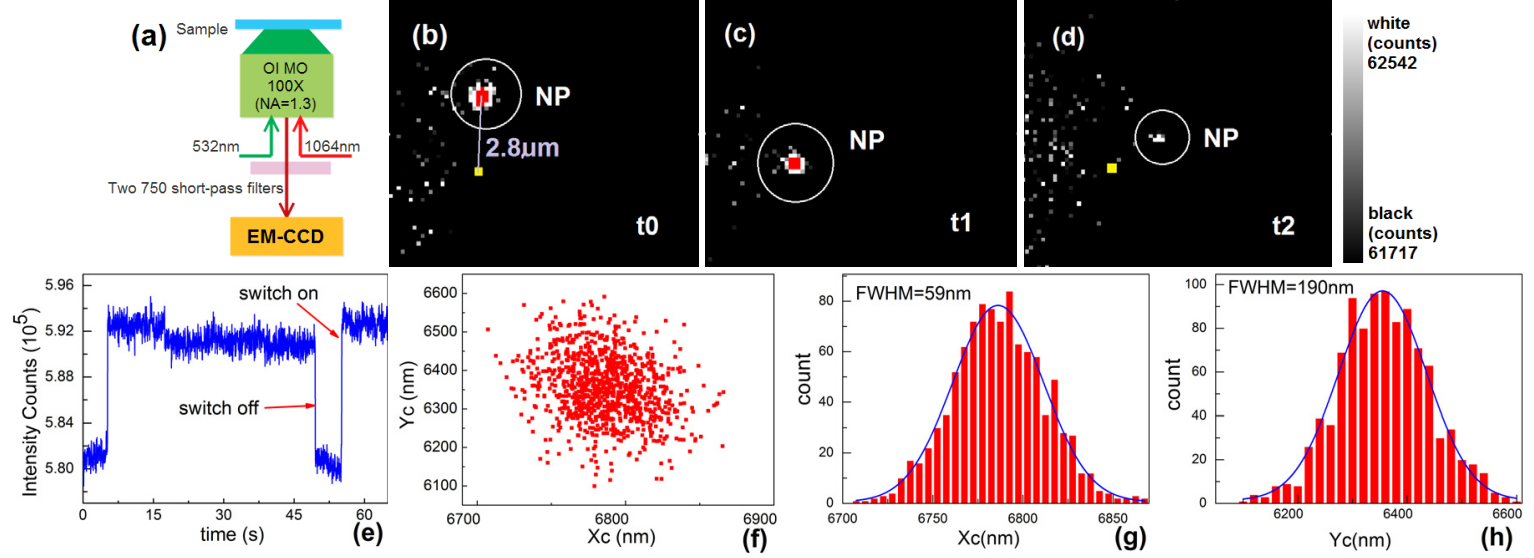

Fig.2. (a) Experimental set-up. (b)-(d) EM-CCD frames showing trapping of NP by DNH (approx. position: yellow dot) and brightness scale bar. $\mathrm{P}=38 \mathrm{~mW}$ (before objective). (e) Fluorescence intensity vs time. $\mathrm{P}=51 \mathrm{~mW}$ (before objective). (f) Scatter plots of NP center locations. $P=15 \mathrm{~m} W$ (before objective). (g),(h) Position histograms along $x$ - and $y$-axes, extracted from data of panel $\mathrm{f}$ ).

\section{References}

[1] M.L. Juan, M. Righini, and R. Quidant. "Plasmon nano-optical tweezers," Nature Photonics 5, 349 (2011)

[2] M.L. Juan, R. Gordon, et al, "Self-induced back-action optical trapping of dielectric nanoparticles." Nature Physics 5, 915 (2009)

[3] A.A. Al Balushi et al, "Label-free free-solution nanoaperture optical tweezers for single molecule protein studies," Analyst 140, 4760 (2015)

[4] L.Neumeier, R.Quidant and D.E Chang, "Self-induced back-action optical trapping in nanophotonic systems," New J. Phys 17, 123008 (2015)

[5] R. Parthasarathy, "Rapid, accurate particle tracking by calculation of radial symmetry centers," Nature Methods 9, 724 (2012) 